\title{
Effect of Nanomicelle Curcumin on Quality of Life and Sleep in Patients With Parkinson's Disease: A Double-Blind, Randomized, and Placebo- Controlled Trial
}

\author{
Mehdi Maghbooli $^{*(\mathbb{D}}$, Bahareh Safarnejad ${ }^{1}$, Hossein Mostafavi $^{\circledR}{ }^{\mathbb{D}}$, Saeideh Mazloomzadeh $^{(\mathbb{D}}$, Abdoreza \\ Ghoreishi' ${ }^{(D)}$ \\ 'Department of Neurology, Zanjan University of Medical Sciences, Zanjan, Iran \\ ${ }^{2}$ Department of Physiology, School of Medicine, Zanjan University of Medical Sciences, Zanjan, Iran \\ ${ }^{3}$ Department of Health Research Center, Zanjan University of Medical Sciences, Zanjan, Iran
}

\begin{abstract}
Background: Considering the evidence indicating the neuronal protective effects of curcumin in previous studies, this double-blind, randomized, placebo-controlled, and parallel-group trial was aimed at exploring the possible nanomicelle curcumin (SinaCurcumin ${ }$, nano-micellar soft gel)mediated impact on sleep, fatigue, and quality of life (QoL) in patients with Parkinson's disease (PD). Methods: A sample of 50 PD patients were recruited and randomly divided into experimental (25) and control groups (25). Sleep quality, fatigue, and QoL were assessed based on the Pittsburgh Sleep Quality Index (PSQI), Fatigue Severity Scale (FSS), and the Parkinson's Disease Questionnaire-39 (PDQ-39), respectively, at the beginning and the end of the study. The groups were treated for three months by $80 \mathrm{mg}$ of nano-micellar soft gel twice a day.

Results: Nanomicelle curcumin significantly increased sleep quality and QoL compared with placebo ( $P$ values $=0.0001$ and 0.0002 , respectively) in PD patients. This significant difference has not influenced by the duration of the disease, the severity of disease progression (Hoehn \& Yahr scale), and the cumulative dose of levodopa. This supplement did not have a significant effect on the fatigue severity of patients compared to placebo.

Conclusion: It has proposed that the nanomicelle curcumin can be used to improve sleep quality and QoL in PD patients.

Keywords: Parkinson's disease; Nanomicelle curcumin; Sleep quality; Fatigue severity; Quality of life.
\end{abstract}

*Correspondence to Mehdi Maghbooli, M.D., Department of Neurology, Zanjan University of Medical Sciences, Zanjan, 4515777978 Iran. Tel: +989144910131 +982433770751 Email:

m.maghbooli@zums.ac.ir

Published online September 23, 2019

Citation: Maghbooli M, Safarnejad B, Mostafavi H, Mazloomzadeh S, Ghoreishi A. Effect of nanomicelle curcumin on quality of life and sleep in patients with Parkinson's disease: a double-blind, randomized, and placebo-controlled trial. Int Clin Neurosci J. 2019;6(4):140145. doi:10.15171/icnj.2019.26.

\section{Introduction}

Parkinson's disease (PD) is a chronic degenerative disease of the nervous system that affects about $1 \%$ of people over the age of 65; after Alzheimer's disease, it is the second most common disease in this group. This disease is associated with the loss of dopaminergic neurons in the dense portion of the substantial nigra in the midbrain and corpus striatum, which results in motor function impairments and, in progressive cases, leads to cognitive impairments.

Previously, the focus was on studying the diseases and their mortality rate, while in recent years, researchers have increasingly focused on studying patients' quality of life $(\mathrm{QoL})$ and health and functional status. ${ }^{1,2}$

In PD, the patients' QoL can improve by identifying factors that reduce QoL (e.g., poor sleep quality, chronic fatigue, and positive interventions). ${ }^{3}$ Common treatments for PD include medication and deep brain stimulation (DBS). However, these therapies only reduce the patient's motor symptoms; that is to say, over time and by the disease progression, their effects also decrease and they may no longer be able to prevent the progressive degeneration of dopaminergic neurons. Long-term use of these drugs also causes some complications, including dyskinesia. ${ }^{4}$ In the treatment process of this complex disease, complementary therapies are also proposed to repair degenerations and reduce symptoms. In this regard, some studies have shown the medical effects of the turmeric plant. They have proved the anti-oxidant, anti-inflammatory, and neuronal protective properties of curcumin. ${ }^{5}$ Modification of dopamine and acetylcholine receptors in the brain of mice with $\mathrm{PD}$ and Alzheimer's

(C) 2019 The Author(s). This is an open access article distributed under the terms of the Creative Commons Attribution License (http:// creativecommons.org/licenses/by/4.0/), which permits unrestricted use, distribution, and reproduction in any medium, provided the original work is properly cited. 
diseases is also one of the beneficial effects attributed to curcumin. The positive effects on cognitive functions and depression are also other features associated with curcumin. ${ }^{6-12}$

One of the important limitations of curcumin is its low bioavailability, which is due to its low solubility in the aquatic environment of the body. ${ }^{1}$ Alzheimer's clinical studies have also shown no significant effects of curcumin, which is due, in part, to its low bioavailability and solubility. ${ }^{11,14}$ The solution to this deficiency, curcumin supplied in other ways, such as in combination with other drugs and the nanoform administration of curcumin, which, on the one hand, increases the bioavailability of this substance and, on the other hand, increases its efficacy. ${ }^{15-17}$ Therefore, this study was set to examine the clinical effects of nanomicelle curcumin on sleep quality, fatigue, and QoL in PD patients.

\section{Methods}

Patients

A double-blind, randomized, placebo-controlled, and parallel-group trial was conducted to determine the efficacy of nanomicelle curcumin (SinaCurcumin ${ }^{\circledR}$. Nano-Micellar Soft gel; Eksir Nano-Sina Minoo Co., Iran) compared with placebo on sleep quality, fatigue, and QoL in PD patients. A total of 50 patients who referred to the Neurology clinic at Vali-e-Asr hospital in Zanjan were selected and randomly divided into experimental and control groups. Inclusion criteria were adapted to include patients with idiopathic PD (MDS-PD criteria); having at least elementary education; filling up the consent form and Unified Parkinson's Disease Rating Scale (Hoehn \& Yahr) (I-III); not changing the drug and supplement therapy since the month prior to the start of the experiment; being 35 years old and over; having no report of allergy to turmeric and other foods and supplements; and having PD history of less than 10 years. Individuals with abnormal renal and liver function tests, cognitive disorders, epilepsy, stroke, and other cerebral diseases, severe disability caused by dementia or other diseases, and gastric disorders, gastroesophageal reflux disease, and gastric ulcers excluded from the study.

\section{Study design}

Restricted randomization was used applying the random allocation rule. Therefore, a sample of 50 participants was selected and randomly assigned into two groups (there were 25 participants in group A and 25 in group B). One group received the drug, and the other one received placebo, but none of the patients knew which group received the drug or placebo. Moreover, all physicians, nurses, statistician, and the person responsible for drafting the manuscript did not know anything about the drug or placebo. SAS software used for randomization. Moreover, sequentially numbered, opaque, sealed envelopes used for allocation concealment.
In the experimental group, $\mathrm{PD}$ patients received one $80 \mathrm{mg}$ soft gelatin capsule of nanomicelle Sina-Curcumin after breakfast and one after dinner for 3 months. The drug contained curcumin (diferuloylmethane), a polyphenol from the group of diarylheptanoids. The most active part of the root of the turmeric plant belongs to the ginger family, which together with the other 2 main uurcuminoids, demethoxycurcumin and bisdemethoxycurcumin, makes up about $3 \%$ to $5 \%$ of the turmeric plant. In the control group, $\mathrm{PD}$ patients received one $80 \mathrm{mg}$ soft gelatin capsule of placebo nanomicelle Sina-Curcumin after breakfast and one after dinner for 3 months; the placebo did not contain any active ingredient. Moreover, the packaging and dosage forms were kept identical.

\section{Outcome Measures}

The data collected via clinical examinations, interviews, and questionnaires before and after the administration of the supplement. Primary data included age, gender, duration of the disease (time elapsed since diagnosis), the severity of the disease, the type of prescribed drug, and the cumulative dose of levodopa. Measurement tools used were Hoehn \& Yahr scale for stage of disease progression, Sleep quality based on the Pittsburgh Sleep Quality Index (PSQI), ${ }^{18}$ Fatigue based on Fatigue Severity Scale (FSS) ${ }^{19}$ and QoL based on the Parkinson's Disease Questionnaire-39 (PDQ-39) ${ }^{20}$ at the beginning and end of the study. The validity and reliability of the Persian version of these questionnaires were previously approved. ${ }^{19,21}$

\section{Statistical Analyses}

Kolmogorov-Smirnov and Shapiro-Wilks tests were used to check for normality of the distribution. All parameters obtained at the beginning and end of the study in the nanomicelle curcumin and placebo groups were compared using paired-samples $t$ test. Mixed-effects regression models have also used to compare the changes in outcome variables before and after intervention with and without possible confounders. All the two-sided $P$ values $<0.05$ were considered significant. SPSS 17 software was used to analyze the data.

\section{Results}

Demographic and Clinical Data

Of the 135 registered patients, 85 were ineligible, not meeting the inclusion criteria. Therefore, 25 patients in the nanomicelle curcumin arm and 25 patients in the placebo arm were eligible (Figure 1). Fifty patients with PD, including 13 women (26\%) and 37 men (74\%), participated in the study. There were no lost-to-follow-up cases in this clinical trial. The mean age of the patients was $65.44 \pm 10.48$. The disease progression stage based on the Hoehn and Yahr scale was $24 \%$ in 12 patients (stage I), $42 \%$ in 21 patients (stage II), and $34 \%$ in 17 patients (stage III). Out of 49 patients, 22 (44\%) took levodopa (L-3,4- 


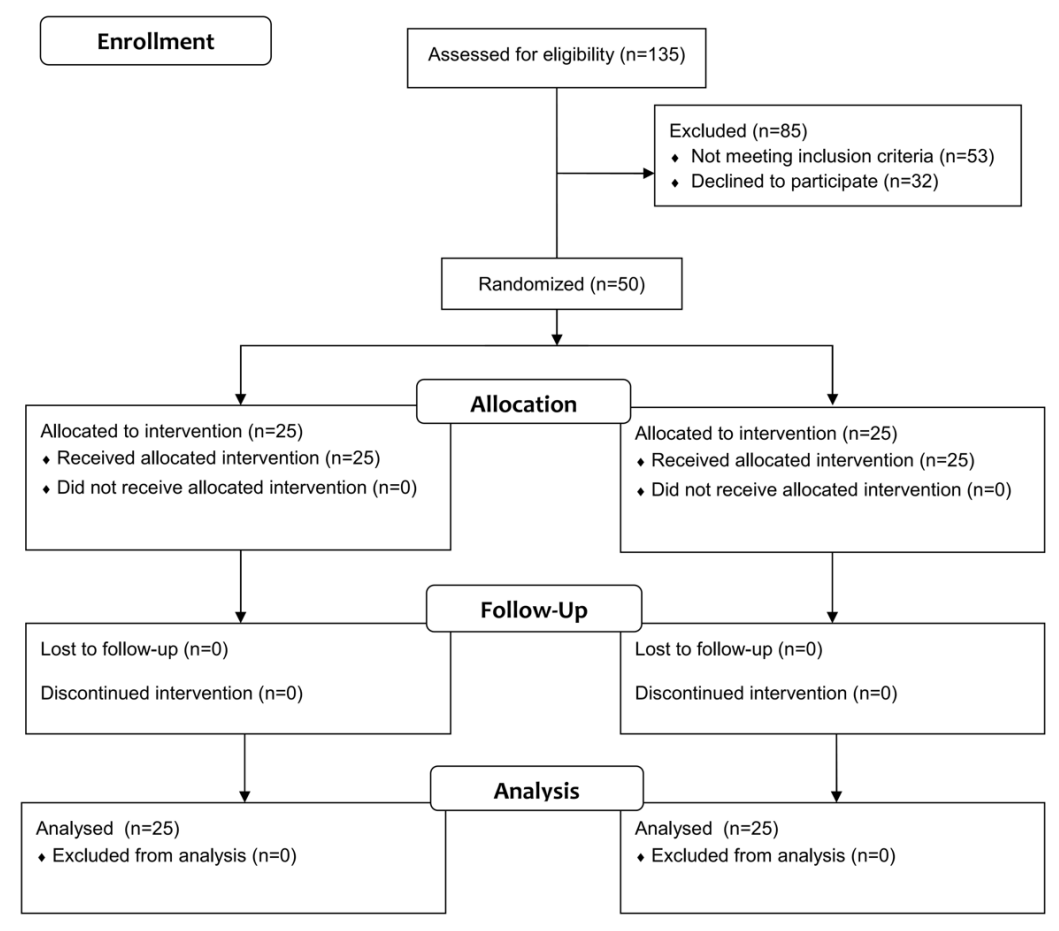

Figure 1. Flowchart of the Study.

dihydroxyphenylalanine-LD), 5 (10\%) took dopamine agonist-DA, and 22 (44\%) took LD and DA. One patient (2\%) received no medication due to the mildness of the symptoms. The levodopa dose was 100 to $499 \mathrm{mg}$ for 20 patients (40\%) and 500 to $1000 \mathrm{mg}$ for 24 patients (48\%). The disease duration varied from 1 to 10 years. It was five years or less in 35 (70\%) patients and more than five years in $15(30 \%)$ patients. Demographic and clinical data presented in Table 1.

\section{Outcome Measures}

The distribution of data in all groups was normal $(P>$ 0.05). The mean values of sleep quality scores based on PSQI in the placebo group before and after the intervention were 9.49 and 7.64, respectively, and in the experimental group were 12.20 and 6.04, respectively. Based on pairedsamples $t$ test, the difference between the mean scores of the 2 groups was significant $(P=0.0001)$ (Table 2$)$. This significant relationship has not influenced by the duration of the disease. The severity of the disease, the cumulative dose of levodopa, since the $P$ value for these variables was higher than 0.05 . However, the relationship has influenced the type of prescribed drug $(P=0.009)$.

Mean values of FSS in the placebo group before and after intervention were 38.92 and 40.60 , respectively, and in the experimental group were 44.40 and 40.16 , respectively. Based on the paired-samples $t$ test, the difference between the mean scores of the two groups was not significant $(P=0.42)$ (Table 2).

The mean scores of QoL based on PDQ-39 in the placebo group before and after the intervention were 36.40 and 34.92, respectively, and in the experimental group were 51.24 and 31.88 , respectively. Based on the paired-samples $t$ test, the difference in mean scores between the two groups was significant $(P=0.0002)$ (Table 2). This significant relationship has not influenced by the duration of the disease, severity, and the cumulative dose of levodopa since the $P$ value for these variables was higher than 0.05 . However, the relationship influenced by the type of prescribed drug $(P=0.02)$.

\section{Discussion}

Nanomicelle curcumin significantly increased the sleep quality and QoL in patients with PD compared to the placebo group ( $P$ values were 0.0001 and 0.0002 , respectively). This correlation has not influenced by the duration of the disease, the severity of the disease, and the cumulative dose of levodopa; nevertheless, the type of prescribed drug-affected it. This supplement did not have a significant effect on the fatigue severity of patients compared with the placebo.

In a randomized polysomnography double-blind study, controlled with placebo, Pierantozzi et al investigated the effectiveness of rotigotine versus placebo regarding PSG parameters in advanced PD patients. For all participants, polysomnography, Parkinson's Disease Sleep Scale (PDSS), PSQI, and motor skill assessment questionnaires administered early in the morning. Fortytwo patients with PD (stages 2 and 3 of H\&Y) and sleep disorders randomly divided into 2 groups. Rotigotine 
Table 1. Demographic and Clinical Data

\begin{tabular}{lcc}
\hline & Nanomicelle Curcumin Group $(\mathbf{n = 2 5})$ & $\begin{array}{c}\text { Placebo Group (n=25) } \\
\text { (Mean } \pm \text { SD) }\end{array}$ \\
\hline Age & (Mean \pm SD) & $63.08 \pm 10.47$ \\
The duration of the disease $(\mathrm{y})$ & $67.80 \pm 10.14$ & $4.68 \pm 3.11$ \\
The cumulative dose of levodopa $(\mathrm{mg} / \mathrm{d})$ & $4.36 \pm 2.37$ & $526.25 \pm 249.27$ \\
\hline
\end{tabular}

Table 2. Comparison of Mean Scores of Sleep Quality Based on PSQI, Fatigue Severity Based on FSS, and QoL Based on PDQ-39 in 2 Selected Groups in PD Patients Using T test for 2 Paired Samples.

\begin{tabular}{|c|c|c|c|c|}
\hline & \multicolumn{2}{|c|}{ Nanomicelle Curcumin Group (N=25) } & \multicolumn{2}{|c|}{ Placebo Group $(\mathrm{N}=25)$} \\
\hline & $\begin{array}{l}\text { Before Intervention } \\
\quad(\text { Mean } \pm S D)\end{array}$ & $\begin{array}{l}\text { After Intervention } \\
\quad(\text { Mean } \pm \text { SD })\end{array}$ & $\begin{array}{l}\text { Before Intervention } \\
\quad(\text { Mean } \pm S D)\end{array}$ & $\begin{array}{l}\text { After Intervention } \\
\quad(\text { Mean } \pm \text { SD })\end{array}$ \\
\hline PSQI & $12.20 \pm 7.79$ & $6.04 \pm 4.42^{\mathrm{a}}$ & $9.40 \pm 7.58$ & $7.64 \pm 6.62$ \\
\hline FSS & $44.40 \pm 17.77$ & $40.16 \pm 14.90$ & $38.92 \pm 17.83$ & $40.60 \pm 14.44$ \\
\hline PDQ-39 & $51.24 \pm 31.31$ & $31.88 \pm 19.36^{b}$ & $36.40 \pm 24.75$ & $34.92 \pm 23.09$ \\
\hline
\end{tabular}

a $P=0.0001 ;{ }^{\text {b } P}=0.0002$

significantly improved sleep efficiency and reduced early morning awakening and sleep latency rates compared to the placebo. Also, mean changes in rapid eye movement sleep rate in the rotigotine group were significantly higher than those in the placebo group. Improvement of PSG parameters was associated with improvement of PDSS symptoms, and PSQI was associated with improvement of early-morning motor symptoms. ${ }^{22}$ Although in the study conducted by Pierantozzi et al rotigotine was used as a dopamine receptor agonist from the non-ergoline drug category, its effectiveness was comparable to that of nanomicelle curcumin supplement in our study.

Chronic fatigue syndrome, infection, and oxidative stress are interrelated in epidemiological studies. However, data demonstrating scientific validation of epidemiological claims regarding the effectiveness of food supplements on chronic fatigue syndrome are lacking. Gupta et al examined the effect of curcumin in a mouse model of immunologically induced fatigue and used pure lipopolysaccharide and Brucella abortus antigens as immunogens. Chronic fatigue syndrome was evaluated based on a chronic periodic stress test for 19 days during immobility times as a fatigue marker. Mice challenged with lipopolysaccharide or Brucella abortus for 19 days; they showed a significant increase in immobility and hyperalgesia on day 19, and their TNF-alpha serum levels increased. Simultaneous treatment with curcumin resulted in a significant reduction in immobility and hyperalgesia. Oxidative stress and TNF-alpha decreased. These findings strongly suggest a significant increase in oxidative stress during immunological activation and confirm that curcumin can be a valuable option in the treatment of chronic fatigue syndrome. ${ }^{23}$ Huang et al evaluated the effect of a curcumin supplement on physiological fatigue and physical performance in rats. The rats were divided into 4 groups and received oral administration of $0,12.3$,
24.6, or $61.5 \mathrm{~mL} / \mathrm{kg} / \mathrm{d}(180 \mu \mathrm{g} / \mathrm{mL})$ curcumin supplement for four weeks. By examining forelimb grip strength, levels of physical fatigue-associated biomarkers, serum lactate, ammonia, blood urea nitrogen, glucose markers, and tissue damage markers such as aspartate transaminase, alanine transaminase, and creatine kinase the anti-fatigue performance evaluated. Curcumin supplement increased grip strength dose-dependently and significantly reduced the amount of lactate, ammonia, blood urea nitrogen, aspartate aminotransferase, alanine aminotransferase, and creatine kinase after a physical challenge. Muscle glycogen content has significantly increased. ${ }^{2}$ Moreover, Ledda et al evaluated the effectiveness of Merami ${ }^{\circledR}$ (CurcuminPhytosome $^{\circledR}$ ) supplement in transient primary renal microalbuminuria. Patients received three capsules per day containing $300 \mathrm{mg}$ of curcumin in a form with a high absorption level.

In comparison with the control group, albuminuria improved within four weeks, and fatigue has significantly decreased or disappeared. ${ }^{25}$ Although these studies indicate the effect of curcumin on the treatment of chronic fatigue syndrome, this supplement did not have a significant effect on the fatigue severity of PD patients in the present study. This difference in outcome may explain by differences in the type of study design and type of diseases.

Pharmaceutical delivery systems that can provide nonbound curcuminoids are essential because the absorption of bioactive and penetrable forms plays a critical role in its effectiveness. Pandaren Sudheeran et al examined the immunity, antioxidant, and bioavailability effectiveness of CurQfen (curcumagalactomannoside (CGM)), a natural formulation of curcumin with fenugreek diet fiber. In this randomized, double-blind trial with a control group receiving placebo, 60 participants with anxiety and fatigue related to occupational stress were randomly assigned to 
receive CGM, standard curcumin, and placebo for 30 days (500 mg twice daily). The participants examined the Medical Outcomes Study Short Form (SF)-36, stress scale, and Beck anxiety scores. A significant improvement in QoL $(P<0.05)$ and significant reductions regarding stress $(P<0.001)$, anxiety $(P<0.001)$, and fatigue $(P<0.001)$ observed in the CGM group as compared with the group receiving standard curcumin. Improvement of QoL was associated with a significant increase in antioxidant markers and reduction of lipid peroxidation. ${ }^{26}$ In this study, $500 \mathrm{mg}$ of CurQfen was used twice a day for a month, while in our study, $80 \mathrm{mg}$ of nanomicelle curcumin (the consumption dose reduced 6 times) was used twice a day for three months. Although the studied groups were different, nanomicelle curcumin significantly increased QoL in PD patients.

Major depression is a common and chronic disease that decreases QoL and increases mortality risk. In a clinical meta-analysis study, Al-Karawi et al examined the role of curcumin in patients with major depressive disorders. Six clinical trials selected according to inclusion criteria. In general, oral administration of curcumin showed a significant reduction in depression symptoms. The subgroup analyses showed that curcumin had the most significant effect on the middle-aged population in long-term use and higher doses. The result showed that curcumin reduces the symptoms of depression in patients with depression. ${ }^{6}$ Although the study conducted by AlKarawi et al was on non-Parkinson groups, their findings were consistent with the results of the current work in terms of the effectiveness of curcumin on QoL.

In sum, we propose that Nanomicelle curcumin may improve sleep quality and QoL in PD patients. The present study has some strengths. It is a double-blind, randomized, placebo-controlled, and parallel-group trial. We used nanomicelle curcumin that has a high absorption rate and is stable in the acidic environment of the stomach for 6 hours. The effect of curcumin on sleep, fatigue, and QoL in PD patients evaluated for the first time based on PDQ-39, PSQI, and FSS questionnaires.

Moreover, other variables such as duration of the disease, severity of the disease, cumulative dose of levodopa, and the type of prescribed drug have also controlled. The limitation of the study may be a relatively low number of participants in each group. On the other hand, the study had a short-term follow-up, and therefore, conclusions are reasonable only for that period of follow-up.

\section{Conflict of Interest Disclosures}

The authors declare that they have no conflict of interests.

\section{Ethical Statement}

The Ethics Committee approved the study of Zanjan University of Medical Sciences (ZUMS.REC.1396.249) based on the ethical guidelines of human research and experimentation expressed in the 1975 Declaration of Helsinki and registered at the Iranian Registry of Clinical Trials (identifier: IRCT20101209005352N2; https:// en.irct.ir/trial/5726). All participants signed the consent forms.

\section{Acknowledgments}

This study supported by the Research Council of the Zanjan University of Medical Sciences. There was no additional external funding received for this study. The supporter had no role in study design, data collection, and analysis, the decision to publish, or preparation of the manuscript.

\section{References}

1. Kalia LV, Lang AE. Parkinson's disease. Lancet. 2015;386(9996):896-912. doi: 10.1016/s01406736(14)61393-3.

2. Sveinbjornsdottir S. The clinical symptoms of Parkinson's disease. J Neurochem. 2016;139 Suppl 1:318-24. doi: 10.1111/jnc.13691.

3. Opara JA, Brola W, Leonardi M, Blaszczyk B. Quality of life in Parkinson's disease. J Med Life. 2012;5(4):375-81.

4. Stoker TB, Torsney KM, Barker RA. Emerging Treatment Approaches for Parkinson's Disease. Front Neurosci. 2018;12:693. doi: 10.3389/fnins.2018.00693.

5. Cole GM, Teter B, Frautschy SA. Neuroprotective effects of curcumin. Adv Exp Med Biol. 2007;595:197-212. doi: 10.1007/978-0-387-46401-5_8.

6. Al-Karawi D, Al Mamoori DA, Tayyar Y. The Role of Curcumin Administration in Patients with Major Depressive Disorder: Mini Meta-Analysis of Clinical Trials. Phytother Res. 2016;30(2):175-83. doi: 10.1002/ptr.5524.

7. Bitu Pinto N, da Silva Alexandre B, Neves KR, Silva AH, Leal LK, Viana GS. Neuroprotective Properties of the Standardized Extract from Camellia sinensis (Green Tea) and Its Main Bioactive Components, Epicatechin and Epigallocatechin Gallate, in the 6-OHDA Model of Parkinson's Disease. Evid Based Complement Alternat Med. 2015;2015:161092. doi: 10.1155/2015/161092.

8. Dai F, Chen WF, Zhou B, Yang L, Liu ZL. Antioxidative effects of curcumin and its analogues against the free-radicalinduced peroxidation of linoleic acid in micelles. Phytother Res. 2009;23(9):1220-8. doi: 10.1002/ptr.2517.

9. Darvesh AS, Carroll RT, Bishayee A, Novotny NA, Geldenhuys WJ, Van der Schyf CJ. Curcumin and neurodegenerative diseases: a perspective. Expert Opin Investig Drugs. 2012;21(8):1123-40. doi: 10.1517/13543784.2012.693479.

10. Daverey A, Agrawal SK. Curcumin alleviates oxidative stress and mitochondrial dysfunction in astrocytes. Neuroscience. 2016;333:92-103. doi: 10.1016/j.neuroscience.2016.07.012.

11. Goozee KG, Shah TM, Sohrabi HR, Rainey-Smith SR, Brown $B$, Verdile G, et al. Examining the potential clinical value of curcumin in the prevention and diagnosis of Alzheimer's disease. $\mathrm{Br} J$ Nutr. 2016;115(3):449-65. doi: 10.1017/ s0007114515004687.

12. Kumar TP, Antony S, Gireesh G, George N, Paulose CS. Curcumin modulates dopaminergic receptor, CREB and phospholipase $\mathrm{C}$ gene expression in the cerebral cortex and cerebellum of streptozotocin induced diabetic rats. J Biomed Sci. 2010;17:43. doi: 10.1186/1423-0127-17-43.

13. Aggarwal BB, Sung B. Pharmacological basis for the role of curcumin in chronic diseases: an age-old spice with modern targets. Trends Pharmacol Sci. 2009;30(2):85-94. doi: 10.1016/j.tips.2008.11.002.

14. Hamaguchi T, Ono K, Yamada M. REVIEW: Curcumin and Alzheimer's disease. CNS Neurosci Ther. 2010;16(5):285-97. doi: 10.1111/j.1755-5949.2010.00147.x. 
15. Kanai M, Imaizumi A, Otsuka $Y$, Sasaki H, Hashiguchi $M$, Tsujiko K, et al. Dose-escalation and pharmacokinetic study of nanoparticle curcumin, a potential anticancer agent with improved bioavailability, in healthy human volunteers. Cancer Chemother Pharmacol. 2012;69(1):65-70. doi: 10.1007/ s00280-011-1673-1.

16. Sasaki H, Sunagawa $Y$, Takahashi K, Imaizumi A, Fukuda $\mathrm{H}$, Hashimoto $\mathrm{T}$, et al. Innovative preparation of curcumin for improved oral bioavailability. Biol Pharm Bull. 2011;34(5):660-5. doi: 10.1248/bpb.34.660.

17. Sunagawa $Y$, Hirano $S$, Katanasaka $Y$, Miyazaki $Y$, Funamoto $\mathrm{M}$, Okamura $\mathrm{N}$, et al. Colloidal submicron-particle curcumin exhibits high absorption efficiency-a double-blind, 3-way crossover study. J Nutr Sci Vitaminol (Tokyo). 2015;61(1):3744. doi: 10.3177/jnsv.61.37.

18. Buysse DJ, Reynolds CF 3rd, Monk TH, Berman SR, Kupfer DJ. The Pittsburgh Sleep Quality Index: a new instrument for psychiatric practice and research. Psychiatry Res. 1989;28(2):193-213. doi: 10.1016/0165-1781(89)90047-4.

19. Fereshtehnejad SM, Hadizadeh H, Farhadi F, Shahidi GA, Delbari A, Lokk J. Reliability and validity of the persian version of the fatigue severity scale in idiopathic Parkinson's disease patients. Parkinsons Dis. 2013;2013:935429. doi: 10.1155/2013/935429.

20. Peto V, Jenkinson C, Fitzpatrick R, Greenhall R. The development and validation of a short measure of functioning and well being for individuals with Parkinson's disease. Qual Life Res. 1995;4(3):241-8.

21. Nojomi M, Mostafavian Z, Shahidi GA, Jenkinson C. Quality of life in patients with Parkinson's disease: Translation and psychometric evaluation of the Iranian version of PDQ-39. J Res Med Sci. 2010;15(2):63-9.

22. Pierantozzi $M$, Placidi F, Liguori $C$, Albanese $M$, Imbriani $P$, Marciani MG, et al. Rotigotine may improve sleep architecture in Parkinson's disease: a double-blind, randomized, placebocontrolled polysomnographic study. Sleep Med. 2016;21:1404. doi: 10.1016/j.sleep.2016.01.016.

23. Gupta A, Vij G, Sharma S, Tirkey N, Rishi P, Chopra K. Curcumin, a polyphenolic antioxidant, attenuates chronic fatigue syndrome in murine water immersion stress model. Immunobiology. 2009;214(1):33-9. doi: 10.1016/j. imbio.2008.04.003.

24. Huang WC, Chiu WC, Chuang HL, Tang DW, Lee ZM, Wei $\mathrm{L}$, et al. Effect of curcumin supplementation on physiological fatigue and physical performance in mice. Nutrients. 2015;7(2):905-21. doi: 10.3390/nu7020905.

25. Ledda A, Belcaro G, Feragalli B, Hosoi M, Cacchio $M$, Luzzi R, et al. Temporary kidney dysfunction: Robuvit(R) supplementation in initial, transient kidney insufficiency and micro-macro albuminuria. Panminerva Med. 2018;60(2):604. doi: 10.23736/s0031-0808.18.03420-1.

26. Pandaran Sudheeran S, Jacob D, Natinga Mulakal J, Gopinathan Nair G, Maliakel A, Maliakel B, et al. Safety, Tolerance, and Enhanced Efficacy of a Bioavailable Formulation of Curcumin With Fenugreek Dietary Fiber on Occupational Stress: A Randomized, Double-Blind, Placebo-Controlled Pilot Study. J Clin Psychopharmacol. 2016;36(3):236-43. doi: 10.1097/ jcp.0000000000000508. 